\title{
Minimum Quality Standards and Predatory Behaviour*
}

\author{
Luca Lambertini - Carlo Scarpa \\ Dipartimento di Scienze Economiche \\ Università di Bologna \\ Piazza Scaravilli 2 \\ 40126 Bologna, Italy \\ e-mail: lamberti@spbo.unibo.it \\ e-mail: cscarpa@economia.unibo.it
}

January 15, 1999

\begin{abstract}
This paper shows that the introduction of a minimum quality standard can have repercussions on market structure, opening the possibility of predatory behaviour. The predatory equilibrium exists independently of whether or not adjustment costs are present. Moreover, whenever predation is an equilibrium, it is selected by the risk dominance criterion.
\end{abstract}

JEL classification: L12, L13

Keywords: MQS, vertical differentiation, predatory behaviour

* Some of the points developed in this paper emerged from comments by Massimo Motta on previous work by one of the authors, and we are grateful for his contribution. We thank Pierpaolo Battigalli for a useful discussion of some of the results. 


\section{Introduction}

The (limited) empirical evidence on the effects of minimum quality standards (MQS) is seemingly at odd with most of the theoretical results in duopoly, which typically point to the desirability of MQS and to a qualityimproving effect of their introduction ${ }^{1}$. For instance, Carroll and Gaston (1991) - analysing the regulation of electricians - indicate that licensing restrictions reduces supply ${ }^{2}$ and decreases average quality (increase accident rates). More recent work on this topic by Chipty and Witte (1997) points in the same direction, showing how minimum standards increase the probability that a firm leaves the market. Furthermore, they observe that the maximum quality supplied may decline after the intoduction of the MQS, whenever the MQS induces some firm to leave the market.

The ideas that emerge from these papers is that the introduction of a MQS affects market structure, in particular inducing exit by some firm, and that this decrease in competition can be associated to quality reductions. In this paper we present a model where these effects are present, showing that the introduction of a MQS can provide a basis for predatory behavior by the high quality firm, and that the likelihood that the MQS reduces supply is substantially higher than indicated, for instance, by the seminal paper by Ronnen (1991).

Previous literature in duopolistic markets, including Donnenfeld and Weber (1992 and 1995), Crampes and Hollander (1995), Constantatos and Perrakis (1997 and 1998), Ecchia and Lambertini (1997), Lutz (1997), is typically based on two assumptions, which are unnecessarily restrictive and that we try to relax in the current paper.

The first one is that, following the introduction of the MQS, the high quality firm will play the duopoly equilibrium strategy, and will not try to drive the rival out of the market. This possibility clearly exists, as the introduction of an MQS limits the ability of the low quality firm to differentiate its output from the rival's. It is sometimes claimed that this seriously undermines the conclusions of previous models, but a formal analysis is still lacking.

Secondly, we want to relax the assumption that adapting to the new MQS (and to other firms' new quality choices) entails no adjustment costs. We feel

\footnotetext{
${ }^{1}$ This is true of most models considering duopolistic markets (starting from Ronnen, 1991), while Scarpa (1998) obtains a different result with three firms.

${ }^{2}$ The supply reducing effect is confirmed, for instance, by Gormley (1991).
} 
this is potentially relevant as the structure of the multi-stage game requires logically the quality choice to be a "long" term choice, that constrains the firm for a significant period of time. Therefore, models without adjustment costs can hardly provide a coherent representation of how incumbent firms react to the new standard ${ }^{3}$.

Within this framework, we show that the duopoly survives only for very low levels of the MQS. For higher values, predatory behaviour is indeed possible, and within a certain interval two equilibria exist: the duopoly equilibrium and a "predatory" one, where the high quality firm reduces its quality level and the rival leaves the market.

When both equilibria exist, we use the criterion of risk-dominance (Harsanyi and Selten, 1988) in order to select the "most likely" equilibrium. This criterion allows us to rule out the duopoly equilibrium for the entire admissible range of parameters. We then show that the same result obtains if we introduce the (more plausible) assumption that shifting quality levels entails some adjustment cost; in this case, the duopoly equilibrium proves even more fragile.

The remainder of the paper is organised as follows. The setup is laid out in section 2. Predatory behaviour when qualities can be adjusted costlessly is described in section 3 . The incentive to predate in the presence of adjustment costs is investigated in section 4 . Section 5 contains concluding remarks.

\section{The model}

We consider a vertically differentiated duopoly, where firms $H$ and $L$ produce goods of either high $(H)$ or low $(L)$ quality. If $q$ denotes quality, firm $i$ 's cost is $C_{i}=C\left(q_{i}\right)$, with $C^{\prime}, C^{\prime \prime}>0$ for all $q_{i} \in[0, \infty), i=H, L$.

A unit mass of consumers indexed by a taste parameter $\theta$ is uniformly distributed over the interval $[0, \Theta]$. Marginal consumers are identified by $\theta_{H}=$ $\left(p_{H}-p_{L}\right) /\left(q^{L F}-q_{L}\right)$ and $\theta_{L}=p_{L} / q_{L}$. Therefore, market demands are

$$
x_{H}=\Theta-\frac{p_{H}-p_{L}}{q_{H}-q_{L}} \text { and } x_{L}=\frac{p_{H}-p_{L}}{q_{H}-q_{L}}-\frac{p_{L}}{q_{L}}
$$

and the profit function of firm $i$ is $p_{i} x_{i}\left(q_{i}, q_{j}\right)-C\left(q_{i}\right)$.

\footnotetext{
${ }^{3}$ This does not mean that previous models are incorrect. Simply, they compare different, alternative scenarios (with and without MQS, or with MQS of different sizes) but not the change in behaviour of a firm and of an industry after the introduction of the MQS.
} 
We only analyse a subgame perfect equilibria where firms choose quality levels first, and then prices. In an unregulated equilibrium, firms produce different quality levels in order to soften price competition (Gabszewicz and Thisse, 1979; Shaked and Sutton, 1982); we denote these levels by $q_{H}^{U}$ and $q_{L}^{U}$. The effects of a minimum quality standard $(s)$ have been studied by Ronnen (1991), who proves that - for some value $S$ - if $s \in\left[q_{L}^{U}, S\right]$ we have that

- an equilibrium exist, such that both firms survive (for $s>S$, the low quality firm leaves the market);

- in this equilibrium, both quality levels are higher than the unregulated ones;

- social welfare is larger;

- the high quality seller's profit is lower;

- furthermore, a value $S^{\prime}$ exists, such that if $s \leq S^{\prime}<S$, the low quality seller's profit increases.

Here we first want to show that there exist values of $s \in\left[q_{L}^{U} ; S\right]$ where a second equilibrium exists, in which the high quality firm forces its rival out of the market and all the above conclusions are reversed ${ }^{4}$.

If a MQS is introduced and both firms survive, firm $i$ will produce quality ${ }^{5}$ $q_{i}(s) \geq s$. Given that prices are chosen optimally, equilibrium profits will be

$$
\begin{gathered}
\pi_{H}=\frac{4 \Theta^{2}\left[q_{H}(s)\right]^{2}\left(q_{H}(s)-q_{L}(s)\right)}{\left(4 q_{H}(s)-q_{L}(s)\right)^{2}}-C\left[q_{H}(s)\right] \\
\pi_{L}=\frac{\Theta^{2} q_{H}(s)\left(q_{H}(s)-q_{L}(s)\right) q_{L}(s)}{\left(4 q_{H}(s)-q_{L}(s)\right)^{2}}-C\left[q_{L}(s)\right]
\end{gathered}
$$

If the low quality firm produces $q_{L}=s$ and the $H$ firm plays its duopoly best reply $\left[q_{H}^{D}(s)\right]$, the profit levels will be denoted by $\pi_{H}^{D}$ and $\pi_{L}^{D}$, respectively. This is the case usually analysed in the literature.

\footnotetext{
${ }^{4}$ As the fact that predation reduces welfare will come to no surprise, welfare issues are not explicitly analysed.

${ }^{5}$ If the standard is binding, the $L$ firm can either produce a quality level equal to it, or else leapfrog its rival. Therefore, $q_{L}(s) \in\left\{s, q^{L F}\left(q_{H}(s)\right)\right\}$, where $q^{L F}\left(q_{H}(s)\right)>q_{H}(s)$ is the quality level the $L$ firm would choose to optimally leapfrog the rival.
} 
One aspect that the literature typically does not consider is the following. As the reaction function of the low quality firm is not continuous in $q_{L}=s$, the high quality firm may find it profitable to adopt a predatory behaviour rather than the best reply $q_{H}^{D}(s)$. Indeed, a quality level lower than $q_{H}^{D}(s)$ might squeeze the low quality demand to a level that forces the low quality firm to leave the market. On the other hand, if the $H$ firm's quality level is too low, leapfrogging might be profitable, so that predation may no longer be feasible.

Let us denote the predatory quality by $q_{H}^{P}(s)$, yielding a monopoly profit $\pi_{M}^{P}\left(q_{H}^{P}(s)\right)$. Predation requires the $L$ firm to have no option but to leave the market, while the $H$ firm earns profits larger that it would in a duopoly equilibrium. Formally, the necessary and sufficient condition for predation is that the following inequalities are simultaneously satisfied:

$$
\pi_{L}\left(q_{H}^{P}(s), q_{L}(s)\right) \leq 0
$$

and

$$
\pi_{M}^{P}\left(q_{H}^{P}(s)\right) \geq \pi_{H}^{D}\left(q_{H}^{D}(s), s\right)
$$

Let us define $Q^{P}:=\left\{q_{H} \mid \pi_{L}\left(q_{H}(s), q_{L}(s)\right) \leq 0\right.$ and $\left.\pi_{M}^{P}\left(q_{H}(s)\right) \geq \pi_{H}^{D}\left(q_{H}^{D}(s), s\right)\right\}$. This is the set of all predatory quality levels. Two remarks are in order.

- The length of this interval depends on $s$. In particular, depending on the value of $s$, the monopoly quality level without entry threats $\left(q^{M}\right)$ may or may not belong to $Q^{P}$.

- As predation requires a relatively low quality level, unless $q^{M} \in Q^{P}$ predation requires the firm to distort its choice: when $q_{H}=q_{H}^{P} \neq q^{M}$, if the $L$ firm leaves the market $\partial \pi_{H} / \partial q_{H}>0$. This entails a cost for the firm, increasing in the difference between the predatory quality level and $q^{M}$.

It is thus straightforward to prove that

Lemma 1 If $q^{M} \in Q^{P}$, then $q_{H}^{P}=q^{M}$. If $q^{M} \notin Q^{P}$, then $q_{H}^{P}=\sup Q^{P}$.

As long as $q_{L}=s$, predation is preferable to accepting the duopoly whenever (5) is satisfied. Hence, for some admissible values of $s$, we may have two equilibria. It is possible to show that predation is easier, the larger the value of $s$. 
Proposition 1 Given $s \in\left[q_{L}^{U} ; S\right]$, if $q_{H}^{P}(s)$ exists, then $d q_{H}^{P}(s) / d s \geq 0$.

Proof. As long as $q^{M} \in Q^{P}$, the predatory quality does not vary with $s$. Thus, let us consider the case $q^{M} \notin Q^{P}$.

Whenever the MQS is binding, $\partial \pi_{L} / \partial s<0$, for a given level of $q_{H}$. On the other hand, given $q_{L}$, from $(3)$ it can be verified that $\partial \pi_{L} / \partial q_{H}>0$. Notice that $q_{H}^{P}(s)$ is defined as the value such that $\pi_{L}\left(q_{H}^{P}(s), s\right)=0$. Applying the implicit function theorem, we can conclude that in this case

$$
\frac{d q_{H}^{P}}{d s}=-\frac{\partial \pi_{L} / \partial s}{\partial \pi_{L} / \partial q_{H}}>0
$$

When the MQS increases, the space available to the low quality firm shrinks, and thus predation can be achieved with a quality level closer to $q_{M}$. This is clearly preferable for the high quality producer, in that its best reply $q_{H}^{D}(s)$ is larger than $q_{H}^{P}(s)$. Any decrease in $q_{H}$ relative to the best reply entails a "cost", so that predation is indeed made "cheaper" by higher levels of $s$.

Proposition 1 implies that predation - whenever feasible - may be profitable either for all values within $\left[q_{L}^{U} ; S\right]$, or in a connected subset of this interval.

On the other hand, if the $H$ firm decreases its quality level "too much", leapfrogging may be feasible. Denote the profit of the $L$ firm which leapfrogs the predator as $\pi^{L F} \equiv \pi\left(q_{H}^{P} ; q^{L F}\left(q_{H}^{P}\right)\right)$. Then, we derive the following:

Lemma 2 If $\pi_{M}^{P}\left(q_{H}^{P}\right)<\pi_{H}^{D}$, then the unique equilibrium is $\left(q_{H}^{D}(s) ; s\right)$.

Proposition 2 If $\pi_{M}^{P}\left(q_{H}^{P}\right)>\pi_{H}^{D}$, and $\pi^{L F}>0$, then again the unique equilibrium is $\left(q_{H}^{D}(s) ; s\right)$.

If $\pi_{M}^{P}\left(q_{H}^{P}\right)>\pi_{H}^{D}$ and $\pi^{L F}<0$, then two equilibria exist; the first one is, again, $\left(q_{H}^{D}(s) ; s\right)$, while the second is $\left(q_{H}^{P}(s) ; s\right)$.

Proposition 3 There exist values of the standard $\underline{q}$ and $\bar{q}$, with $q_{L}^{U} \leq \underline{q} \leq$ $\bar{q} \leq S$, such that

Corollary $1 \quad \bullet$ for $s \in\left[q_{L}^{U} ; \underline{q}\right)$ when $q_{L}=s$ predation is not profitable;

- for $s \in[\underline{q} ; \bar{q})$ if $q_{H}=q_{H}^{P}$ the best reply of the other firm is to leapfrog $\left(\pi^{L F}\left(q_{H}^{P} ; q^{L F}\left(q_{H}^{P}\right)\right)>0\right)$; 
- for $s \in[\bar{q} ; S]$ predation is profitable.

Proof. Obvious, along the lines of the Proof of Proposition 1.

Therefore, the interval $\left[q_{L}^{U} ; S\right]$ can be divided in three regimes. In the third one, when predation is profitable we have the aforementioned multiplicity and thus we have a potential problem of equilibrium selection, which may be solved with the use of the notion of risk-dominance ${ }^{6}$.

At the final stage, prices are always chosen optimally given quality levels. At the first stage, the strategy space of each firm is binary, with $q_{H} \in\left\{q_{H}^{D}(s), q_{H}^{P}(s)\right\}$ and $q_{L} \in\{s, 0\}$ where $q_{L}=0$ implies that the low quality firm exits the market. The reduced form of the game is described by Matrix 1.

$$
\begin{aligned}
& L
\end{aligned}
$$

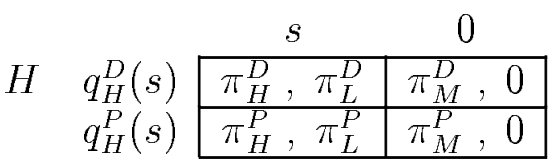

The two Nash equilibria of this game are the duopoly equilibrium $\left(q_{H}^{D}(s)\right.$, $s)$ and the predatory equilibrium $\left(q_{H}^{P}(s), 0\right)$, while $\pi_{M}^{D}$ and $\pi_{i}^{P}$ are out-ofequilibrium payoffs. $\pi_{M}^{D}$ is the monopoly profit that the high quality firm obtains when it produces the best reply to the MQS and the rival exits the market. $\pi_{i}^{P}$ is the duopoly profit for firm $i(i=H, L)$ when the high quality firm produces the predatory quality and the low quality firm sticks to the MQS.

Applying the criterion of risk-dominance to this case we can see that the predatory equilibrium risk-dominates the other one when

$$
\left(\pi_{H}^{D}-\pi_{H}^{P}\right) \pi_{L}^{D}>-\left(\pi_{M}^{P}-\pi_{M}^{D}\right) \pi_{L}^{P}
$$

\footnotetext{
${ }^{6}$ See Harsanyi and Selten (1988). The existing plethora of equilibrium concepts potentially opens numerous - too many - possibilities. The choice of risk-doiminance as a selection criterion is due first of all to the relation between risk-dominance and tremblinghand perfection (Kajii and Morris, 1997), a criterion that in the situation examined seems intuitively appropriate. Notice that an analogous choice was done by Motta et al. (1997).
} 
This inequality is a complex expression in $s$, which may or may not hold within the framework adopted so far. We can investigate this aspect and the actual feasibility of predatory strategies by slightly specialising the model relative to Ronnen.

\section{Predation in the standard model}

Let us consider the model of the previous section, setting ${ }^{7} \Theta=1$ and $C\left(q_{i}\right)=$ $q_{i}^{2}$. Unregulated quality levels are $q_{L}^{U}=0.024119$ and $q_{H}^{U}=0.126655$. As to the behaviour of the regulator in choosing the quality standard $s$, the following holds:

Remark 1 If the high quality firm plays the best response to $s$, the market can remain a duopoly only if $s \leq 0.04809$.

Proof. Solving the game backwards, suppose firms play the Nash equilibrium prices. The best reply function of the high quality firm is

$$
\begin{aligned}
q_{H}\left(q_{L}\right)= & \frac{6 q_{L}+1}{24}+\frac{1-6 q_{L}}{\left.24\left(1-9 q_{L}+324 q_{L}^{2}+3 q_{L} \sqrt{3\left(23-208 q_{L}+3888 q_{L}^{2}\right.}\right)\right)^{1 / 3}}+ \\
& +\frac{\left.\left(1-9 q_{L}+324 q_{L}^{2}+3 q_{L} \sqrt{3\left(23-208 q_{L}+3888 q_{L}^{2}\right.}\right)\right)^{1 / 3}}{24}
\end{aligned}
$$

Plugging (8) into (3), we can see that $\pi_{L}\left(q_{H}^{D}\left(q_{L}\right), q_{L}\right)=0$ at $q_{L}=0.04809$. This establishes that $S=0.04809$.

Define the social welfare function as the sum of profits and consumer surplus, $S W=\sum_{i} \pi_{i}+C S$, where

$$
C S=\int_{\theta_{L}}^{\theta_{H}}\left(\theta q_{L}-p_{L}\right) d \theta+\int_{\theta_{H}}^{1}\left(\theta q_{L}-p_{L}\right) d \theta
$$

\footnotetext{
${ }^{7}$ This is just meant to facilitate the derivation of explicit results. As $\Theta$ increases, it can be shown that $q_{i}(\Theta)=\Theta^{2} q_{i}(\Theta=1)$ and $\pi_{i}(\Theta)=\Theta^{4} \pi_{i}(\Theta=1)$. For a proof, see Motta (1993).
} 
Plugging (8) into the welfare function, it is easily verified that social welfare is increasing and concave for all $q_{L} \in[0.02412,0.06761)$. Therefore, it is easy to show that, absent predation, it is socially optimal to set $s=0.04809$.

As for the possibility of predation, a preliminary step consists in calculating the monopoly optimum (without threats). From (1), the demand function is $x=1-p / q$, where $p / q$ is the generic price-quality ratio offered by the monopolist. Accordingly, the monopolist's profit function is $\pi^{M}=p x-q^{2}$. Given the monopoly price $p^{M}=q / 2$, the profit function simplifies to $\pi^{M}=q(1 / 4-q)$, which is maximised at $q^{M}=1 / 8$.

Consider now predatory behaviour by the high-quality firm. The condition $\pi_{L}=0$ is yields

$$
\hat{q}_{H}=\frac{q_{L}\left[\left(8 q_{L}-1\right)-\sqrt{1-12 q_{L}}\right]}{2\left(16 q_{L}-1\right)} .
$$

As stated in Lemma 1, whenever $q^{M} \leq \widehat{q}_{H}$, then $q_{H}^{P}=q^{M}$. Otherwise, given a generic MQS $s$, this defines the predatory quality level: $q_{H}^{P}=\hat{q}_{H}$. Numerical calculations show that $q^{M}=\widehat{q}_{H}$ at $q_{L}=0.047366$; therefore, we have established that, without leapfrogging,

$$
\begin{gathered}
q_{H}^{P}=\frac{s[(8 s-1)-\sqrt{1-12 s}]}{2(16 s-1)} \forall s \in(0.02412,0.04737] \\
q_{H}^{P}=q^{M}=\frac{1}{8} \forall s \in[0.04737,0.04809] .
\end{gathered}
$$

It is fairly obvious that monopoly profits are larger than $\pi_{H}^{D}(s)$. This indicates that predation is certainly feasible and profitable for all $s \in(0.04737,0.04809]$. It remains to be established whether, in the remainder of the admissible range for $q_{L}$, the quality level defined by (11) is optimal, i.e. whether $\pi_{M}^{P}\left(q_{H}^{P}(s)\right)>\pi_{H}^{D}\left(q_{H}^{D}(s), s\right)$. Numerical calculation reveals that

$$
\pi_{M}^{P}>\pi_{H}^{D}\left(q_{H}^{D}(s), s\right) \quad \forall s \in(0.03356,0.04737],
$$

and conversely for all $s \in(0.02412,0.03356]^{8}$. Therefore:

Remark 2 Whenever $s \leq 0.03356$, predation is not profitable.

\footnotetext{
${ }^{8}$ When $s=0.033556$, the corresponding predatory quality is $q_{H}^{P}=0.054503$.
} 
This indicates that for very low levels of $s$, predation would require the high quality firm to distort its choice by "too much", and would thus be too costly. In the previous notation, this means that $\underline{q}=0.03356$.

So far we have assumed that $q_{L}=s$. Let us now focus on the possibility for the low-quality firm to leapfrog the rival, if the latter tries to predate.

With $q^{L F}\left(q_{H}^{P}\right)>q_{H}^{P}$, the profit from leapfrogging is:

$$
\pi^{L F}=\frac{4\left[q^{L F}\left(q_{H}^{P}\right)\right]^{2}\left(q^{L F}\left(q_{H}^{P}\right)-q_{H}^{P}\right)}{\left(4 q^{L F}\left(q_{H}^{P}\right)-q_{H}^{P}\right)^{2}}-\left[q^{L F}\left(q_{H}^{P}\right)\right]^{2},
$$

with $q^{L F}\left(q_{H}^{P}\right)>q_{H}^{P}, q_{H}^{P}=\min \left\{\hat{q}_{H}, q^{M}\right\}$. Evaluating (14) for all $q_{H}^{P} \in$ $[0.054503,1 / 8]$, reveals that

$$
\pi^{L F}\left(q^{L F}\left(q_{H}^{P}\right) ; q_{H}^{P}\right)>0 \quad \forall q_{H}^{P} \in[0.054503,1 / 12) .
$$

Moreover, $q_{H}^{P}=1 / 12$ when $s=0.04117$. Therefore, we can state

Remark 3 Given $s \in(0.03356,0.04117)$, any $q_{H}=q_{H}^{P}(s)$ makes leapfrogging profitable.

To sum up, a low level of the MQS $(s \leq 0.03356)$ makes predation unprofitable even if the low quality firm sticks to $q_{L}=s$. A slightly higher level of $s$ allows predation only if the low quality firm keeps producing the minimum quality level, but this is not rational. Indeed, in the interval indicated in Remark 3, if the $H$ firm assumes that the rival produces $q_{L}=s$ and in turn produces the correspondent "predatory" quality level, the (previously) low quality firm will not produce $s$, but will leapfrog the rival. Therefore, in this case predation will not take place in equilibrium. This leads to the final conclusion

Proposition 4 Whenever $s \in[0.04117,0.04809]$ an equilibrium exists, in which the high quality firm produces $q_{H}=q_{H}^{P}(s)$, forcing the rival to exit the market.

When the minimum quality standard is set within this interval, the high quality firm will produce $q_{H}^{P}$ and the low quality firm will not be able to obtain positive profits either producing $q_{L}=s$ or leapfrogging the rival. Given that the high quality firm adopts a predatory behaviour, the market is too small for two firms to coexist. 
Notice that, in the interval of Proposition 4, a second equilibrium exists, in which $q_{L}=s$ and $q_{H}=q_{H}^{D}(s)$. In order to select one equilibrium, we can use the criterion of risk dominance, already introduced in (7). Plugging: expressions $(8),(10),(12)$ and (11) into (7), we obtain an extremely complex expression in $s$, which can be evaluated numerically to find that $(7)$ is satisfied for all $s \in[0.04117 ; 0.04809]$. This establishes the final result of this section:

Proposition 5 Whenever two equilibria exist, the criterion of risk dominance selects the equilibrium with predation.

This result is hardly surprising, given the analogy between risk dominance and trembling-hand perfection (see Kajii and Morris, 1997). If the $H$ firm produces the predatory quality and the $L$ firm insists in supplying. the minimum quality standard, the latter firm is going to suffer substantial losses, while its potential gain (the duopoly profit $\pi_{L}^{D}$ ) is anyway sufficiently close to zero.

\section{Predation with adjustment costs}

Unlike most standard models of MQS, here we want to analyse a situation in which the introduction of a standard hits an already existing market, where firms produce certain quality levels, and have to adjust their products accordingly. This need is certainly present for a low quality firm, whose product in the unregulated equilibrium is "too poor", but the change in the low quality product induces an analogous response by the high quality rival.

We thus consider a two stage situation. In the first, unregulated period firms produce $q_{H}^{U}$ and $q_{L}^{U}$, defined above. In the second period, firms not only pay the current production cost $C\left(q_{i}\right)=q_{i}^{2}$, but also an adjustment cost that we assume to be quadratic in the difference between the new quality and the one previously produced:

$$
C^{a}\left(q_{i}\right)=q_{i}^{2}+\left(q_{i}-q_{i}^{U}\right)^{2}
$$

We now replicate the analysis carried out in the previous section, under the assumption that the cost function is (16). The best reply of firm $H$ is implicitly defined by

$$
\frac{\partial \pi_{H}}{\partial q_{H}}=2 q_{H}^{U}-4 q_{H}+\frac{4 q_{H}\left[4 q_{H}^{2}-3 q_{H} q_{L}+2 q_{L}^{2}\right]}{\left(4 q_{H}-q_{L}\right)^{3}}=0
$$


It is easy to check that when $q_{L}(s)=s=0.04214$ and $q_{H}^{D}(s)=0.12858$, $\partial \pi_{H} / \partial q_{H}=0$ and $\pi_{L}=0$ while social welfare is still increasing in $s$. We can thus derive the following:

Remark 4 If the high quality firm plays the best response to $s$, the market can remain a duopoly only if $s \leq 0.04214$.

Not surprisingly, the maximum level of $s$ compatible with duopoly is lower than the one without adjustment costs.

Let us now turn to predatory behaviour. The general condition for $\pi_{L}=0$ - which defines a candidate predatory quality - is

$$
\begin{aligned}
\widehat{q}_{H}^{a}= & q_{L} \frac{5.1658 \cdot 10^{9}-1.54 \cdot 10^{12} q_{L}+1.78 \cdot 10^{13} q_{L}^{2}}{2\left(1.033 \cdot 10^{10}-1.97 \cdot 10^{12} q_{L}+3.557 \cdot 10^{13} q_{L}^{2}\right)}- \\
& q_{L} \frac{\left(77824-8.6 \cdot 10^{21} q_{L}+1.95 \cdot 10^{24} q_{L}^{2}-2.96 \cdot 10^{25} q_{L}^{3}+2.75 \cdot 10^{11} q_{L}^{4}\right)^{1 / 2}}{2\left(1.033 \cdot 10^{10}-1.97 \cdot 10^{12} q_{L}+3.557 \cdot 10^{13} q_{L}^{2}\right)}
\end{aligned}
$$

It remains true that, whenever $q^{M} \leq \hat{q}_{H}^{a}$, then $q_{H}^{P}=q^{M}$. Numerical calculations show that $q^{M}=\widehat{q}_{H}^{a}$ when $q_{L}=0.04193$; therefore, assuming $q_{L}=s$, we have established that

$$
\begin{gathered}
q_{H}^{P}=\widehat{q}_{H}^{a} \forall s \in(0.02412,0.04193] ; \\
q_{H}^{P}=q^{M}=\frac{1}{8} \forall s \in[0.04193,0.04214] .
\end{gathered}
$$

Notice that the role of adjustment costs is somehow ambiguous. On the one hand, adapting its quality to the standard becomes more costly for firm $L$ and therefore its profit goes to zero more rapidly as $s$ increases. On the other one, if the $H$ firm wants to adopt a predatory strategy, it has to change its quality level, and this is also more costly in the presence of adjustment costs.

As monopoly profits are larger than duopoly profits, predation is profitable for all $s \in(0.04193,0.04214)$. Replicating the previous analysis, we can see that

$$
\pi_{M}^{P}>\pi_{H}^{D}\left(q_{H}^{D}(s), s\right) \quad \forall s \in(0.03661,0.04193],
$$

and conversely for all $s \in(0.02412,0.03661]$. Therefore: 
Remark 5 Whenever $s \leq 0.03661$, predation is not profitable.

Again, the comparison with the previous case is the most interesting aspect. Like before, we have a lower limit on the standard, that might make predation profitable. However, now this limit is higher than without adjustment costs, because their presence makes it more costly to decrease quality. If $s \in(0.03356 ; 0.03661)$, predation is profitable in the absence of adjustment costs, while now it does not lead to profits large enough to justify the quality change.

As for leapfrogging, it is easy to show that given our assumption on adjustment costs this strategy does not pay off. Whenever $q_{H}=q_{H}^{P}$ as defined by (19) and (20), there is no value of $q_{L}>q_{H}^{P}$ such that the $L$ firm earns positive profits. Therefore, with adjustment costs whenever predation is profitable, it is also feasible (leapfrogging is not a best reply for the $L$ firm). Therefore, we have established the following result:

Proposition 6 In the presence of adjustment costs as specified in (16), whenever $s \in[0.03661 ; 0.04214)$ an equilibrium exists, in which the high quality firm produces $q_{H}=q_{H}^{P}(s)$ and the rival exits the market.

Obviously, as before, whenever predation is possible we can have two equilibria, one with predation and a duopoly equilibrium. The same selection problem analysed in the previous section arises. Again, we can see that predation is not always the risk-dominant equilibrium.

To this end, we have replaced the relevant values of quality levels in expression (7) and we obtain an expression in $q_{L}$ that numerical analysis shows to be satisfied for all $s \in[0.03661 ; 0.04214]$. We thus have our last result:

Proposition 7 In the presence of adjustment costs as in (16), the criterion of risk dominance always selects the equilibrium with predation.

The interpretation of this result is analogous to the one of the previous section. Now the length of the interval where the duopoly equilibrium obtains is substantially smaller than in the case without adjustment costs ${ }^{9}$. The cost the $L$ firm must pay when $s$ is large is obviously larger than without adjustment costs, and this makes its survival more problematic.

\footnotetext{
${ }^{9}$ One might argue that adjustment costs are asymmetric, i.e., have to be paid in order to increase quality, while worsening one's product is always possible at zero cost. It is possible to show that the same qualitative conclusions hold in this case.
} 


\section{Concluding remarks}

While the initial literature on minimum quality standards tended to stress the positive welfare effects of such regulatory interventions, this paper, in line with a growing theoretical and empirical literature, points in the opposite direction. The introduction of an MQS can have repercussions on market structure, opening the possibility of predatory behaviour. We have shown that the predatory equilibrium exists independently of whether or not adjustment costs are present. Moreover, whenever predation is an equilibrium, it is selected by the risk dominance criterion.

Although some of the basic assumptions of the standard model we have adopted look quite restrictive, we do not feel that any of our results are particularly model-specific. Probably, the most relevant extension of the present analysis should consider a variable cost technology as in Crampes and Hollander (1995), but we leave this for future research. 


\section{References}

[1] Carroll, S. and R. Gaston (1991), Occupational Restrictions and the Quality of Service Received: Some Evidence, Southern Economic Journal, 47(4), 959-77.

[2] Chipty, T. and A. D. Witte (1997), An Empirical Investigation of Firms' Reponses to Minimum Quality Standards Regulations, NBER Working Paper 6104.

[3] Constantatos, C. and S. Perrakis (1997), Vertical Differentiation: Entry and Market Coverage with Multiproduct Firms, International Journal of Industrial Organization, 16(1), 81-103.

[4] Constantatos, C. and S. Perrakis (1998), Minimum Quality Standards, Entry, and the Timing of the Quality Decision, Journal of Regulatory Economics, 13(1), 47-58.

[5] Crampes, C. and A. Hollander (1995), Duopoly and Quality Standards, European Economic Review; 39(1), 71-82.

[6] Donnenfeld, S. and S. Weber (1992), Vertical Product Differentiation with Entry, International Journal of Industrial Organization, 10(3), 44972 .

[7] Donnenfeld, S. and S. Weber (1992), Limit Qualities and Entry Deterrence, RAND Journal of Economics, 26(1), 113-30.

[8] Ecchia, G. and L. Lambertini (1997), Minimum Quality Standards and Collusion, Journal of Industrial Economics, 45(1), 101-13.

[9] Gabszewicz, J.J. and J.-F. Thisse (1979), Price Competition, Quality and Income Disparities, Journal of Economic Theory, 20(3), 340-59.

[10] Gormley, W. (1991), State Regulations and the Availability of Child Care Services, Journal of Policy Analysis and Management, 10(1), 7895.

[11] Harsanyi, J.C. and R. Selten (1988), A General Theory of Equilibrium in Games, Cambridge, MA, Harvard University Press. 
[12] Kajii, A. and S. Morris (1997), The Robustness of Equilibria to Incomplete Information, Econometrica, 65(6), 1283-1309.

[13] Lutz, S. (1997), Vertical Product Differentiation and Entry Deterrence, Journal of Economics, 65(1), 79-102.

[14] Motta, M. (1993), Endogenous Quality Choice: Price vs Quantity Competition, Journal of Industrial Economics, 41(2), 113-32.

[15] Motta, M, Thisse J.-F. and A. Cabrales (1997), On the Persistence of Leadership or Leapfrogging in International Trade, International Economic Review, 38(4), 809-24.

[16] Ronnen, U. (1991), Minimum Quality Standards, Fixed Costs, and Competition, RAND Journal of Economics, 22(4), 490-504.

[17] Scarpa, C. (1998), Minimum Quality Standards with More than Two Firms, International Journal of Industrial Organization, 16(5), 665-76.

[18] Shaked, A. and J. Sutton (1982), Relaxing Price Competition through Product Differentiation, Review of Economic Studies, 49(1), 3-13. 